\title{
Extra-articular tenosynovial chondromatosis of the left ring finger in a 23-year-old man: A case report and literature review
}

\author{
YU-XIAN CHEN*, YUN-XIANG LU*, ZE ZHUANG and ZHI-YONG LI \\ Department of Joint Surgery and Orthopedic Trauma, The Third Affiliated Hospital, \\ Sun Yat-Sen University, Guangzhou, Guangdong 510630, P.R. China
}

Received September 15, 2014; Accepted July 27, 2015

DOI: $10.3892 /$ etm.2015.2687

\begin{abstract}
Tenosynovial chondromatosis is an extra-articular version of articular synovial chondromatosis and a relatively rare condition that can affect the tendon sheath, bursa, or joint synovial tissue. Tenosynovial chondromatosis is rarely reported in the literature and is often misdiagnosed. In the present study, a case of extra-articular tenosynovial chondromatosis of the left ring finger in a 23-year-old man is reported. Three different-sized nodules were identified upon surgery and all were removed via synovectomy. The patient was symptom free 6 months postoperatively, and there were no signs of recurrence after 1.5 years of follow-up. The literature describing tenosynovial chondromatosis in the fingers is also reviewed.
\end{abstract}

\section{Introduction}

Primary synovial chondromatosis is a rare, benign, proliferative cartilaginous lesion that can arise from the tendon sheath, bursa or joint synovial tissue $(1,2)$. Tenosynovial chondromatosis is an extra-articular version of articular synovial chondromatosis, and it is most commonly observed in the fingers and feet. Tenosynovial chondromatosis has rarely been reported in the publicly available literature and is often erroneously classified as a cartilage or soft tissue tumor. In addition, numerous patients with tenosynovial chondromatosis are asymptomatic, and the histological diagnosis can be challenging (3). The present study describes a case of tenosynovial chondromatosis in the finger tendon sheath of a 23 -year-old

Correspondence to: Professor Zhi-Yong Li, Department of Joint Surgery and Orthopedic Trauma, The Third Affiliated Hospital, Sun Yat-Sen University, 600 Tianhe Road, Guangzhou, Guangdong 510630, P.R. China

E-mail: jointsurgry@163.com

*Contributed equally

Key words: tenosynovial chondromatosis, finger, tendon sheath synovia male patient. The literature associated with tenosynovial chondromatosis affecting the fingers is also reviewed.

\section{Case report}

A 23-year-old male patient was admitted to the Third Affiliated Hospital of Sun Yat-Sen University (Guangzhou, China) with a history of pain and progressive swelling at the proximal interphalangeal joint of the left ring finger for the past 2 years. The symptoms and decreased range of motion of the metacarpophalangeal joint were greatly affecting the patient's daily life in the weeks leading up to his admission. The patient recalled no history of trauma or overuse of the left hand. A physical examination of the finger revealed an $\sim 3 \times 2 \mathrm{~cm}$ irregular mass at the volar aspect of the ring finger. The mass had a hard texture, clear boundary and mild tenderness with pressure. The left ring finger metacarpophalangeal joint had a decreased range of motion of $60^{\circ}$. Blood biochemistry for the patient was unremarkable. Preoperative ultrasonography of the left ring finger revealed multiple nodules with well-defined boundaries in the tendon sheath. Since chondromatosis rarely occurs extra-articularly, the nodules were initially and preliminarily diagnosed as a chondroma of the soft tissue parts.

Surgical resection of the nodules was performed. Under brachial plexus anesthesia, a 'Z'-shaped incision was made at the volar aspect of the left ring finger proximal phalanx to expose the subcutaneous tumor. Three different-sized nodules were excised $(2.0 \times 1.5,1.2 \times 0.6$ and $0.7 \times 0.3 \mathrm{~cm})$ during surgery. The nodules were encapsulated loose white bodies in the flexor tendon sheath, with a hard texture and smooth surface (Figs. 1 and 2). Histopathological examination of the nodules revealed increased chondrocyte cellularity and a fibrous capsule, consistent with a diagnosis of tenosynovial chondromatosis (Fig. 3). The patient was symptom free 6 months postoperatively. At a follow-up 1 year after that, there were no signs of recurrence.

\section{Discussion}

Synovial chondromatosis is a rare condition characterized by the formation of multiple cartilaginous nodules in the synovial membrane. Synovial cells are similar to mesenchymal stem cells and are capable of giving rise to cartilage or bone tissue under specific conditions (4). Synovial chondromatosis can 
involve the joints (articular synovial chondromatosis) and the tendon sheath (tenosynovial chondromatosis). While both intra-articular and extra-articular synovial chondromatosis are histologically very similar, they are distinct entities with regard to surgical strategies and recurrence rate (5).

Various names have been given to the nodules found in the tendon sheath, including synovial chondromatosis, synovial cartilage metaplasia, tenosynovial osteochondroma, cartilage and soft tissue benign tumors, and soft tissue tumors. A previous study proposed that all these diagnoses were essentially tenosynovial chondromatosis (6). The proper diagnosis of tenosynovial chondromatosis is clinically challenging due to its rarity, particularly when no calcification or ossification is present in the tumor $(2,3)$. Secondary synovial chondromatosis is caused by degenerative or traumatic lesions, which lead to the formation of intra-articular cartilage.

In 1977, Milgram described synovial chondromatosis as occurring in the following three distinct phases: i) active intrasynovial disease with no free loose bodies; ii) transitional lesions with osteochondral nodules in the synovial membrane and osteochondral bodies lying free within the joint cavity; and iii) multiple free osteochondral bodies with quiescent intrasynovial disease $(5,7)$.

Tenosynovial chondromatosis most frequently affects the hands and feet (8). A total of 26 cases of tenosynovial chondromatosis in the fingers, including the present case, have been documented in the literature $(1-4,6,8,9)$. These cases include 14 males and 13 females, ranging from 30 to 60 years of age. The right hand (16 cases) was more frequently affected than the left hand ( 8 cases). In the two remaining cases, there was one incidence of bilateral disease, and 1 case where the affected hand was not specified. There have been 5 cases of tenosynovial chondromatosis in the thumb, 3 cases affecting the index finger, 9 cases affecting the middle finger, and 5 cases affecting the ring or pinky finger. The flexor tendon was involved in all of the reported cases, but the extensor tendon was never involved. Patients with tenosynovial chondromatosis usually present with no history of trauma. The two most common symptoms are painless swelling that can occur over several months to several years and mild tenderness when pressure is applied. Many patients are asymptomatic, while others develop a trigger finger deformity or carpal tunnel syndrome. Due to the atypical clinical manifestations and the slow disease process, clinical treatment for tenosynovial chondromatosis is often delayed $(3,4,9)$.

Plain radiography of tenosynovial chondromatosis may show extra-articular soft tissue swelling with multiple small calcifications or ossifications. A large calcified body with clusters may suggest a lobular mass $(3,5,7)$. Synovial swelling may lead to bone corrosion, though a periosteal reaction is rarely observed. In patients with a long history of tenosynovial chondromatosis, round, oval or elongated mineralizations can be seen within the tendon sheath, although the adjacent joint is usually not affected.

Computed tomography (CT) of tenosynovial chondromatosis can clearly identify the calcified nodules and their exact locations as well as cortical erosion (10). Detection of tenosynovial chondromatosis with magnetic resonance imaging (MRI) depends on the degree to which the nodules have mineralized. In the majority of cases, the nodules display

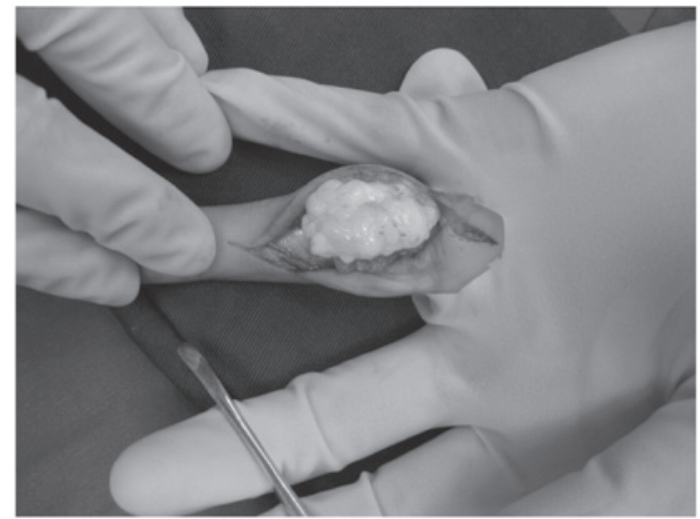

Figure 1. Intraoperative view of the white cartilage-like loose nodule resulting from tenosynovial chondromatosis in the left ring finger.

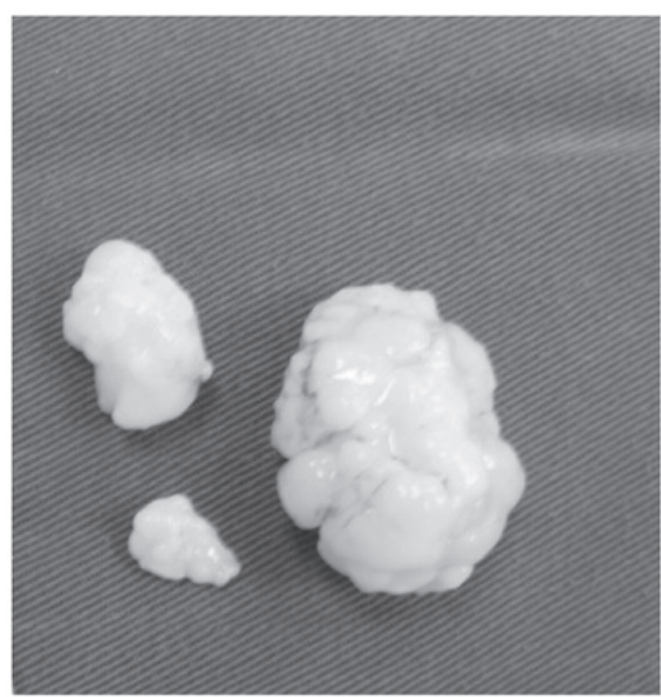

Figure 2. Three white cartilage-like loose bodies were removed from the left ring finger.

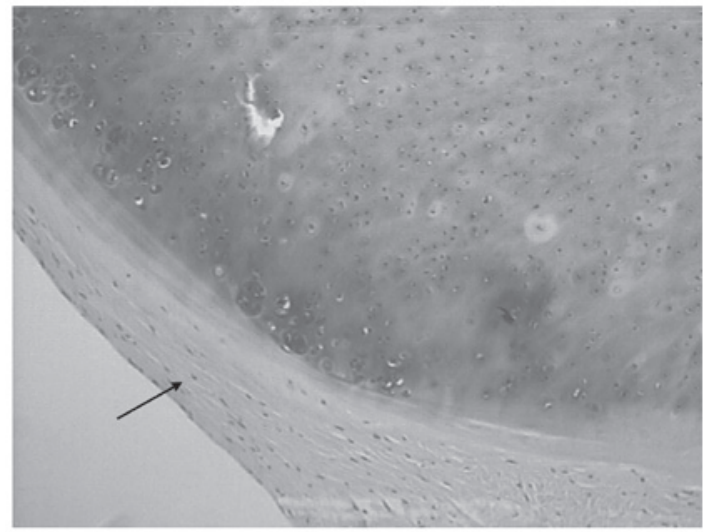

Figure 3. Histopathologic examination of tenosynovial chondromatosis revealed chondrocyte proliferation. Hematoxylin and eosin staining showed that the nodule was covered by a fibrous capsule (black arrow; magnification: $\mathrm{x} 100)$.

a muscle-like signal intensity on T1-weighted images and exhibit high signal intensity on T2-weighted images (11). In the present case, preoperative ultrasonography revealed 
multiple nodules with well-defined boundaries in the tendon sheath. Thus, ultrasonography represents a convenient and cost-effective alternative to CT or MRI for the detection of tenosynovial chondromatosis.

Gross examination of the excised tumor often shows multiple, white, transparent, lobular cartilage nodules, which are easily isolated from the synovial tendon sheath. There can be thousands of nodules of various shapes, ranging in size from a few millimeters to several centimeters, located in the synovial membrane or synovial tendon sheath. In the majority of cases of tenosynovial chondromatosis, closely packed smaller cartilage nodules in the tendon sheath merge into larger nodules $(1,5,6)$. Histopathologic examination may show hyaline cartilage nodules surrounded by synovial membrane. Chondrocytes show mild or moderate atypia. Certain cases show a mucinous change, calcification or ossification, with nodules surrounded by giant cells. As the histologic appearance of tenosynovial chondromatosis is atypical, an erroneous diagnosis of chondrosarcoma can easily be made. However, chondrosarcoma rarely occurs in the hands or feet, a fact that can aid in the differential diagnosis $(1,3,5)$.

Tenosynovial chondromatosis should be differentiated from several other lesions that give rise to bone cartilage formation. Soft tissue tumors should be considered first, as they occur more frequently than synovial chondromatosis and have a much lower recurrence rate. Soft tissue tumors are usually solitary, well-encapsulated, and generally occur in a younger patient population, ranging from 10 to 39 years of age. This disease has rarely been reported to have malignant transformation $(3,5,12)$. Chondrosarcoma should also be considered due to a small risk for malignant transformation. Evidence of aggressive behaviors through imaging, such as cortical erosion or periosteal reaction, is indicative of chondrosarcoma with malignancy. A definitive diagnosis of chondrosarcoma results from histologic findings, as chondrosarcoma predominantly originates from the bone (5). Secondary synovial chondromatosis is usually caused by osteochondral fracture or joint surface exfoliation. Periosteal chondroma mainly occurs in children and young adults and predominantly affects the proximal humerus (6). Other differential diagnoses that can be ruled out by a histologic examination include tenosynovial giant cell tumor, calcifying aponeurotic fibroma, tumoral calcinosis, hydroxy-apatite deposition disease, a foreign body, and inflammatory arthritis.

Tenosynovial chondromatosis is characterized by a slow progression that can last for decades. There are also reports that the nodules can spontaneously be resorbed (12). Malignant chondrosarcoma has been documented in 5\% of 53 cases of synovial chondromatosis (13). By contrast, to the best of our knowledge, no malignant cases of tenosynovial chondromatosis have been reported. Although the association between complete synovial excision and the low relapse rate is not yet clear, most doctors recommend complete removal the loose bodies. Therefore, delicate surgical dissection around the nodules and satellite nodules and a synovectomy are necessary $(3,12)$. In the present case, the patient underwent successful surgical removal of the loose bodies and the surrounding synovial membrane and was followed up for 1.5-years without recurrence.

The reported recurrence rates for tenosynovial chondromatosis vary considerably. In some studies with relatively small sample sizes, no recurrence or a very low recurrence rate was reported. However, one study reported a high recurrence rate of $88 \%$ in a larger cohort of 37 cases of tenosynovial chondromatosis in the hands and feet (6). The recurrence of synovial chondromatosis or tenosynovial chondromatosis can take several months to several years $(4-6,9)$.

The literature reviewed in this study indicate that no available methods are able to apply to the total nature of synovial chondromatosis preoperatively. However, ultrasonography may represent a convenient and cost-effective alternative to CT or MRI imaging for early diagnosis. As for treatment, resection is the preferred method for synovial chondromatosis.

\section{Acknowledgements}

This study was supported by the Science and Technology Program of Guangzhou (No. 2011Y1-00033-4)

\section{References}

1. Khadilakar MS, Patil AA, Shah NS, Deshmukh SD and Anand M: Extra-osseous tenosynovial chondromatosis of the middle finger: A case report. J Orthop Surg (Hong Kong) 20: 406-408, 2012.

2. Gil-Albarova J, Morales-Andaluz J, Castiella T and Seral F: Tenosynovial chondromatosis of the third finger. Arch Orthop Trauma Surg 120: 239-240, 2000.

3. Ueo T, Kashima K, Daa T, Kashima N, Tsuji K, Hisaoka M and Yokoyama S: A case of tenosynovial chondromatosis with tophus-like deposits. APMIS 112: 624-628, 2004.

4. Chan WL, Hung LK, Griffith JF, Louis TC and Ho PC: Tenosynovial osteochondromatosis of both flexor and extensor tendons. Hand Surg 9: 89-95, 2004.

5. Milgram JW: Synovial osteochondromatosis: A histopathological study of thirty cases. J Bone Joint Surg Am 59: 792-801, 1977.

6. Fetsch JF, Vinh TN, Remotti F, Walker EA, Murphey MD and Sweet DE: Tenosynovial (extraarticular) chondromatosis: An analysis of 37 cases of an underrecognized clinicopathologic entity with a strong predilection for the hands and feet and a high local recurrence rate. Am J Surg Pathol 27: 1260-1268, 2003.

7. Cebesoy O, Isik M, Subasi M, Karsli B and Pamukcu U: Extra-articular tenosynovial chondromatosis mimicking a neoplastic disease in the first web space of the hand. Oman Med J 27: 316-318, 2012.

8. DeBenedetti MJ and Schwinn CP: Tenosynovial chondromatosis in the hand. J Bone Joint Surg Am 61: 898-903, 1979.

9. Bui-Mansfield LT, Rohini D and Bagg M: Tenosynovial chondromatosis of the ring finger. AJR Am J Roentgenol 184: 1223-1224, 2005.

10. Ho YY and Choueka J: Synovial chondromatosis of the upper extremity. J Hand Surg Am 38: 804-810, 2013.

11. Murphey MD, Vidal JA, Fanburg-Smith JC and Gajewski DA: Imaging of synovial chondromatosis with radiologic-pathologic correlation. Radiographics 27: 1465-1488, 2007.

12. Christoforou D, Strauss EJ, Abramovici L and Posner MA: Benign extraosseous cartilage tumours of the hand and wrist. J Hand Surg Eur Vol 37: 8-13, 2012.

13. Apte SS and Athanasou NA: An immunohistological study of cartilage and synovium in primary synovial chondromatosis. J Pathol 166: 277-281, 1992. 